\title{
Comparação da distribuição de pressão plantar em sujeitos com pés normais e com pés planos durante a marcha
}

\author{
Comparison of plantar pressure distribution in subjects \\ with normal and flat feet during gait
}

\author{
Patrik Felipe Nazario ${ }^{1}$ \\ João Otacilio Libardoni dos Santos ${ }^{1}$ \\ Aluisio Otavio Vargas Avila ${ }^{1,2}$
}

1. Universidade do Estado de Santa Catarina. Centro de Ciências da Saúde e do Esporte. Laboratório de Biomecânica. Florianópolis, SC. Brasil.

2. Instituto Brasileiro de Tecnologia do Couro, Calçado e Artefatos. Novo Hamburgo, RS. Brasil.

Recebido em 05/10/09 Revisado em 07/02/10 Aprovado em 11/03/10
Resumo - O objetivo do estudo/pesquisa foi verificar possíveis relações entre a queda do arco longitudinal medial, mensurada em situação estática pela altura do osso navicular e um conjunto de variáveis relacionadas à distribuição de pressão plantar, mensuradas em situação dinâmica. Participaram do estudo 11 (onze) sujeitos do gênero masculino, com idade média de 21 anos \pm 3 anos, massa corporal $74 \mathrm{~kg} \pm 10 \mathrm{~kg}$ e estatura $175 \mathrm{~cm} \pm 4 \mathrm{~cm}$. Para aquisição dos dados de Distribuição de Pressão Plantar (Pico de Pressão, Pressão Média, Área de Contato e Carga Relativa), foi utilizado o Novel Emed-AT System com uma taxa de amostragem de $50 \mathrm{~Hz}$ e para análise da Altura do Navicular para classificação dos sujeitos em grupos, foi utilizado o protocolo Navicular Drop Test proposto por Brody (1982). Os dados foram comparados a partir dos resultados da aplicação da estatística não paramétrica através do teste $U$ de Mann-Whitney com $\mathrm{p} \leq 0,05$. Os resultados demonstraram que os grupos foram significativamente diferentes entre si, na região do mediopé, em todas as variáveis analisadas, sendo que foram encontrados os maiores valores médios no grupo dos indivíduos com os Pés Planos. Também apresentaram diferenças significativas na variável Área de Contato, Carga Relativa, Pico de Pressão Plantar e Pressão Plantar Média quando comparados os grupos. Os resultados demonstram a importância que se deve dar aos sujeitos com pés planos, pois alterações na Distribuição de Pressão Plantar estão associadas ao surgimento de desconforto e também de lesões.

Palavras-chave: Arco longitudinal medial; Pé plano; Navicular drop test; Distribuição de pressão plantar.

Abstract - The aim of this study was to determine the possible relationship between loss of the normal medial longitudinal arch measured by the height of the navicular bone in a static situation and variables related to plantar pressure distribution measured in a dynamic situation. Eleven men $(21 \pm 3$ years, $74 \pm 10 \mathrm{~kg}$ and $175 \pm 4 \mathrm{~cm})$ participated in the study. The Novel Emed-AT System was used for the acquisition of plantar pressure distribution data (peak pressure, mean pressure, contact area, and relative load) at a sampling rate of $50 \mathrm{~Hz}$. The navicular drop test proposed by Brody (1982) was used to assess the height of the navicular bone for classification of the subjects. The results were compared by the Mann-Whitney $U$ test, with the level of significance set at $p \leq 0.05$. Differences were observed between the two groups in the mid-foot region for all variables studied, with the observation of higher mean values in subjects with flat feet. There were also significant differences in contact area, relative load, peak pressure, and mean pressure between groups. The present study demonstrates the importance of paying attention to subjects with flat feet because changes in plantar pressure distribution are associated with discomfort and injuries.

Key words: Medial longitudinal arch; Flat foot; Navicular drop test; Plantar pressure distribution. 


\section{INTRODUÇÃO}

Pé plano é o termo utilizado para descrever o tipo de pé que possui um arco longitudinal medial reduzido. Este arco está relacionado com o amortecimento do impacto e transferência da carga para as articulações e exerce uma influência na mecânica funcional do pé1. O pé plano é uma deformidade desenvolvida ou adquirida ${ }^{2}$, e é caracterizado pela rotação plantar medial do tálus, diminuição da altura do arco plantar longitudinal medial e abdução do ante-pés,4. Porém, esse tipo de característica anatômica do pé também pode ser consequência de uma rotação medial fibular e femoral acentuada e ainda a um valgismo de calcâneo ${ }^{5}$. Entretanto, é necessário atenção especial, pela tendência de maior incidência de lesões, dores no joelho, tendinite patelar e fascite plantar em sujeitos com redução do arco plantar.

A diminuição do arco longitudinal medial pode ser observada através da medição da altura do osso navicular, pela possibilidade de descrever e classificar tipos diferentes de pés. Este tipo de medição é utilizada, também, como um bom indicador de parâmetros antropométricos da estrutura do pé e-8 $^{-8}$, por ser considerada como um método não invasivo, válido e confiável para determinar a queda do arco plantar, ${ }^{9}$.

Mens e Morris (2005) afirmam que, em alguns casos, a distribuição das pressões plantares sofre influência e se altera em função da anatomia do pé. Para isso, estudos biomecânicos ${ }^{12-16}$ têm sido importantes para identificação e compreensão dos fatores predisponentes a alterações na distribuição de pressão plantar. Portanto, faz-se necessário estabelecer algumas relações entre parâmetros antropométricos e comportamento da distribuição de pressão plantar, em função da frequente alteração observada na antropometria do pé e a coexistência de altas pressões plantares. Diante do exposto, este estudo objetivou verificar possíveis relações entre a queda do arco longitudinal medial, mensurada em situação estática pela altura do osso navicular e um conjunto de variáveis relacionadas à distribuição de pressão plantar, mensuradas em situação dinâmica.

\section{PROCEDIMENTOS METODOLÓGICOS}

Este estudo descritivo tipo estudo de caso foi realizado no Laboratório de Biomecânica do Centro de Ciências da Saúde e do Esporte (CEFID) da Universidade do Estado de Santa catarina (UDESC).
Participaram do estudo 11 (onze) sujeitos do gênero masculino (21 \pm 3 anos de idade, $74 \pm 10 \mathrm{~kg}$ e 1,75 \pm $0,4 \mathrm{~cm}$ ), que não apresentavam histórico de cirurgia em membros inferiores. Ao chegar ao laboratório de pesquisa, os sujeitos foram informados dos procedimentos e objetivos da coleta e assinaram um termo de consentimento livre e esclarecido aprovado pelo Comitê de Ética e Pesquisa em Seres Humanos da UDESC, sob o protocolo n ${ }^{\circ}$ 184/06.

A coleta de dados foi realizada em duas etapas (Estática e Dinâmica). Na Etapa Estática, os sujeitos foram orientados a sentar-se confortavelmente em uma cadeira com os pés posicionados sobre uma superfície lisa e plana e sem suporte de peso, com um ângulo aproximado de $90^{\circ}$ entre os segmentos da coxa e perna. Assumiu-se que o peso corporal foi distribuido de forma similar na superficie. Após o posicionamento do sujeito, foi determinada a Altura do Osso Navicular. A seguir, solicitou-se que o mesmo levantasse e ficasse na posição ortostática e equilibrasse novamente seu peso corporal homogeneamente em ambos os pés. Após um intervalo de $5 \mathrm{~s}$, a mensuração da Altura do Osso Navicular foi realizada (em pé). Esse procedimento foi baseado no Navicular Drop Test, proposto por Brody ${ }^{17}$, porém o cartão milimetrado foi substituído por um traçador de altura (Mytutoyo - Absolute Digimatic), com uma resolução de 0,01 mm e comprimento de haste de $30 \mathrm{~cm}$. A diferença na Altura do Osso Navicular entre as posições em pé e sentada foi considerada como a altura de queda do osso navicular.

Na Etapa Dinâmica, às variáveis de Distribuição de Pressão Plantar foram coletadas. Os sujeitos foram instruídos a caminhar descalço em uma passarela plana em trajetória retilínea e com uma velocidade de $1,38 \mathrm{~m} / \mathrm{s} \pm 10 \%$, que foi monitorada por duas fotocélulas. Uma plataforma de pressão (Novel Emed-AT) com resolução de 4 sensores capacitivos por $\mathrm{cm}^{2}$ (total de 2736 sensores) do sistema e taxa de amostragem de $50 \mathrm{~Hz}$ foi empregada para determinar os parâmetros relativos à pressão plantar. Após explicação e demonstração da tarefa a ser executada, os sujeitos realizaram 5 repetições válidas, na qual tocaram a superfície da plataforma com todo seu pé direito e na velocidade pré-estabelecida.

As variáveis selecionadas para o estudo foram: Altura do Osso Navicular (altura da borda inferior do ponto mais proeminente medialmente do osso navicular), Área de Contato (área total de contato do pé com o solo), Pico de Pressão Plantar (máxima pressão registrada nas áreas plantares específicas, ou seja, regiões anatômicas selecionadas), Pressão 
Plantar Média (valor médio de pressão representativo de áreas plantares específicas, ou seja, regiões anatômicas selecionadas) e Carga Relativa (relação dos impulsos locais de uma determinada região anatômica e o somatório do impulso de todas as regiões).

Para a obtenção mais detalhada dos dados referentes à Distribuição de Pressão Plantar, foi elaborado um sistema de máscaras no próprio software do sistema Emed- AT. Estas máscaras dividiram o pé em 4 regiões (retropé - M1, mediopé - M2, metatarsos - M3 e dedos - M4) conforme a Figura 1.

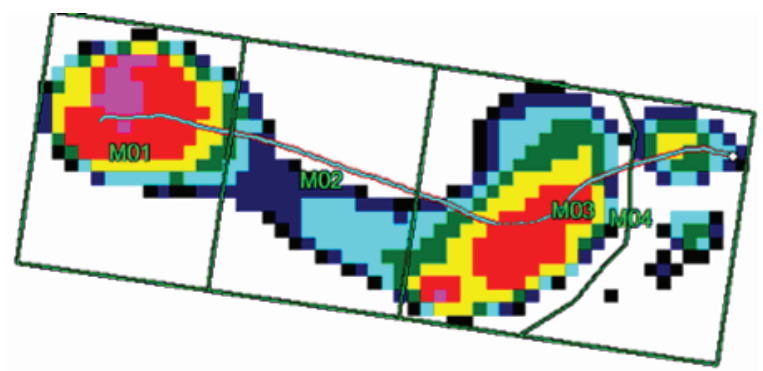

Figura 1: Plantigrama de distribuição de pressão plantar dividido em 4 regiões do pé (M1, M2, M3 e M4).

Os dados obtidos da Distribuição de Pressão Plantar foram processados pelo software da plataforma Emed System e exportados para o software excel. Os dados referentes à Altura do Navicular foram tabulados no excel e divididos em dois grupos: Grupo com Pés Normais (GN, $\mathrm{n}=5$; queda da Altura do Navicular entre $5 \mathrm{~mm}$ - $10 \mathrm{~mm}$ ) e Grupo com Pés Planos (GP, n = 6; queda da Altura do Navicular $\geq 10 \mathrm{~mm}$ ).

O teste de Kolmogorov-Smirnov determinou a falta de normalidade dos dados. Assim, as médias das variáveis entre os grupos foram realizadas por meio do teste U de Mann-Whitney e o nível de significância de $\mathrm{p}<0.05$ foi adotado. As análises foram comparadas no pacote estatístico SPSS 16.0.

\section{RESULTADOS}

A Tabela 1 mostra as variáveis de Distribuição de Pressão Plantar e a comparação, por região do pé, entre os GN e GP. O teste U de Mann-Whitney mostrou que os grupos foram, significativamente, diferentes na região do mediopé, em todas as variáveis analisadas, e os maiores valores foram encontrados no GP. A Área de Contato apresentou valores $7 \%$ maiores na região do mediopé ( $\mathrm{p}$ = 0,0001) no GP, quando comparados com o GN. O Pico de Pressão Plantar e Pressão Plantar Média apresentaram diferenças estatisticamente significativas, na região do mediopé, entre os grupos, sendo que os valores médios do Pico de Pressão Plantar

Tabela 1. Comparação das variáveis cinéticas entre os grupos.

\begin{tabular}{|c|c|c|c|c|c|}
\hline & \multicolumn{2}{|c|}{$\begin{array}{l}\text { Grupo com Pés Normais }(\mathrm{dp}) \\
\qquad \mathrm{n}=5\end{array}$} & \multicolumn{2}{|c|}{$\begin{array}{l}\text { Grupo com Pés Planos (dp) } \\
\qquad n=6\end{array}$} & \multirow[t]{2}{*}{$P$} \\
\hline & Média & D.P. & Média & D.P. & \\
\hline $\begin{array}{l}\text { Área de Contato (\%) } \\
\text { - Retropé } \\
\text { - Mediopé } \\
\text { - Antepé } \\
\text { - Dedos }\end{array}$ & $\begin{array}{l}28,84 \\
14,94 \\
39,45 \\
16,75\end{array}$ & $\begin{array}{l}- \\
- \\
- \\
-\end{array}$ & $\begin{array}{l}25,26 \\
21,60 \\
35,94 \\
17,18\end{array}$ & $\begin{array}{l}- \\
- \\
-\end{array}$ & $\begin{array}{c}0,364 \\
0,0001 * \\
0,326 \\
0,246\end{array}$ \\
\hline $\begin{array}{l}\text { Pico de Pressão Plantar (kPa) } \\
\text { - Retropé } \\
\text { - Mediopé } \\
\text { - Antepé } \\
\text { - Dedos }\end{array}$ & $\begin{array}{c}422,80 \\
88,00 \\
396,80 \\
428,20\end{array}$ & $\begin{array}{c}121,30 \\
23,23 \\
128,79 \\
110,02\end{array}$ & $\begin{array}{l}447,50 \\
111,83 \\
439,33 \\
487,67\end{array}$ & $\begin{array}{c}187,09 \\
18,12 \\
111,28 \\
158,03\end{array}$ & $\begin{array}{c}0,987 \\
0,0001^{*} \\
0,068 \\
0,335\end{array}$ \\
\hline $\begin{array}{l}\text { Carga Relativa }(\%) \\
\text { - Retropé } \\
\text { - Mediopé } \\
\text { - Antepé } \\
\text { - Dedos }\end{array}$ & $\begin{array}{c}31,96 \\
4,66 \\
52,09 \\
11,26\end{array}$ & $\begin{array}{l}3,66 \\
2,48 \\
3,43 \\
3,96\end{array}$ & $\begin{array}{l}28,44 \\
11,44 \\
48,15 \\
11,95\end{array}$ & $\begin{array}{l}4,04 \\
3,98 \\
4,00 \\
2,53\end{array}$ & $\begin{array}{c}0,002^{*} \\
0,001^{*} \\
0,005^{*} \\
0,353\end{array}$ \\
\hline
\end{tabular}

$* p<0,05$ 
na região do mediopé do GP foi 21\% maior que no GN ( $\mathrm{p}=0,0001)$. Na Pressão Plantar Média, observa-se, também, um aumento de $21 \%$ a favor do GP $(p=0,005)$. Houve aumento na Carga Relativa, nas regiões do retropé $(\mathrm{p}=0,002)$ e antepé $(\mathrm{p}=$ 0,005), no GN, em comparação ao GP. Na região do mediopé, observou-se um aumento significativo de, aproximadamente, $7 \%$ no GP, quando comparado ao GN.

\section{DISCUSSÃO}

Ao analisar a Área de Contato nas regiões do pé, percebeu-se um aumento médio de, aproximadamente, $7 \%$ na região do mediopé, nos sujeitos com Pés Planos (21,6\%), quando comparados aos sujeitos com pés normais. Esses achados demonstram que as caracteristicas anatômicas influenciaram no padrão dinâmico da distribuição de pressão plantar, pois os sujeitos com pés planos apresentaram uma maior área de contato na região medial. Esse aumento ocorreu devido à hiper-flexibilidade (depressão) apresentada pelo arco longitudinal medial (característico do pé plano ${ }^{18}$ ), durante a marcha, o que faz com que o pé sustente o peso do corpo em sua face interna. Concordando com nossos resultados, Bacarin et al. ${ }^{15}$ evidenciaram um aumento dos valores médios na área de contato,os quais também foram atribuídos às caracteristicas anatômicas desse tipo de pé.

Como se pode observar, na Tabela 1 , os valores médios do Pico de Pressão Plantar e Pressão Plantar Média na região do mediopé também apresentaram aumentos significativos no grupo que demonstrava alterações nas caracteristicas anatômicas do pé (Pé Plano). Assim, nosso estudo demonstra que essas alterações no padrão dinâmico da distribuição de pressão plantar (Pico Pressão Plantar e Pressão Plantar Média) ocorreram pela influência das alterações na anatômia do pé do grupo analisado (depressão do arco longitudinal medial devido a hiper-flexibilidade e instabilidade do pé plano), o que reflete uma pior condição de distribuição de pressão plantar nesses sujeitos, pois pressões elevadas causam ulcerações e desconforto ${ }^{19-21}$. Entretanto, essas maiores pressões em áreas específicas, conforme apresentado em nosso estudo pelos sujeitos com pés planos, levam à necessidade de maiores cuidados em quadros clínicos, pois o aumento do Pico de Pressão Plantar está relacionado com dores nos pés e com o surgimento de lesões ${ }^{22-24}$, tornando-se ainda mais graves em sujeitos com doenças circulatórias ou diabetes ${ }^{24}$.
Ao analisar a Carga Relativa (Tabela 1), podese observar que os sujeitos do GP apresentaram, na região do mediopé, valores médios $7 \%$ maiores quando comparados com o GN. Esse aumento já era esperado, pois conforme a literatura ${ }^{14}$, sujeitos com pés planos apresentam um aumento na Carga Relativa devido às características anatômicas do pé. Portanto, pode-se destacar que essa variável deve ser considerada uma importante determinante na identificação de possíveis lesões (principlamnete em sujeitos com pés planos por apresentarem um aumento da Carga Relativa), pois o aumento dessa carga na região do mediopé está associado a uma rotação excessiva da tibia sobre o tálus, proporcional ao nível de pronação da articulação subtalar ${ }^{19-21,25}$.

\section{CONCLUSÃO}

É possível concluir com os resultados desse estudo que os indivíduos com alterações nas características anatômicas do pé (depressão do arco longitudinal medial) apresentaram modificações (aumento de intensidade) no padrão dinâmico da distribuição de pressão plantar (Área de Contato, Pico de Pressão Plantar, Pressão Plantar Média e Carga Relativa), principalmente, na região do mediopé. Isso significa que esses sujeitos apresentam riscos maiores a desenvolver ulcerações e desconforto, pois, segundo a literatura, pressões elevadas em áreas específicas levam ao surgimento de lesões. Entretanto, fica cada vez mais clara a importância que se deve dar aos sujeitos com esse tipo de característica (Pé Plano). È importante ressaltar, também, a relevância da análise da distribuição de pressão plantar no quadro clinico e preventivo, pois essa tem um grande potencial para predizer alterações durante a locomoção.

\section{REFERÊNCIAS BIBLIOGRÁFICAS}

1. McCrory JL, Young MJ, Boulton AJM, Cavanagh PR. Arch index as a predictor of arch heigh. The Foot 1997;7:79-81.

2. Williams DS, McClay IS. Measurements used to characterize the foot and the medial longitudinal arch: reliability and validity. Phys Ther 2000;80:864-71.

3. Arangio GA, Reinert KL, Salathe EP. A biomechanical model of the effect of subtalar arthroereisis on the adult flexible flat foot. Clin Biomech 2004;19:847-52.

4. Arangio GA, Salathe EP. Medial displacement calcaneal osteotomy reduces the excess forces in the medial longitudinal arch of the flat foot. Clin Biomech 2001;16:535-9.

5. Cailliet R. Dor no pé e no tornozelo. $3^{\mathrm{a}}$ Ed. Porto 
Alegre: Artmed; 2005.

6. Gilmour JC; Burns Y. The measurement of the medial longitudinal arch in children. Foot Ankle Int 2001;22:493-8;

7. Chia-Hsin CMD, et al. The Correlation between selected measurements from footprint and radiograph of flat foot. Arch Phys Med Rehabil. 2006;87:235-40.

8. Shrader JA, Popovich JM, Gracey GC, Danoff JV. Navicular drop measurement in people with rheumatoid arthritis: interrater and intrarater reliability. Phys Ther 2005;85:656-64.

9. McPoil TG, Cornwall MW. The relationship between static lower extremity measurements and rearfoot motion during walking. J Orthop Sports Phys Ther 1996;24:309-14.

10. Williams DS, McClay IS, Hamill J. Arch structure and injury patterns in runners. Clin Biomech 2001;16:341-7.

11. Menz HB, Morris ME. Clinical determinants of plantar forces and pressures during walking in older people. Gait \& posture 2005;21:72-9.

12. Ledoux WR, Hillstrom HJ. The distributed plantar vertical force of neutrally aligned and pes planus feet. Gait \& Posture 2002;15:01-9.

13. Burns J, Crosbie J, Hunt A, Ouvrier R. The effect of pes cavus on foot pain and plantar pressure. Clin Biomech 2005;20:877-82.

14. Urry SR, Wearing SC. Arch indexes from ink footprints and pressure platforms are different. The Foot. 2005;15:68-73.

15. Bacarin TA, Canettieri MG, Akashi PMH, Sacco ICN. Plantar pressure distribution differences between flat and normal feet in healthy subjects (Abstract). J Biomech 2006;39:111.

16. Putti AB, Arnold GP, Cochrane LA, Abboud RJ. Normal pressure values and repeatability of the Emed ${ }^{\circledR}$ ST4 System. Gait \& Posture 2008;23:501-5.

17. Brody D. Techniques in the evalution and treatment of the injured runner. Orthop Clin North Am 1982;13:542-58.

18. Birtane M, Tuna $H$. The evaluation of plantar pressure distribution in obese and non-obese adults. Clin Biomech 2004;19:1055-9.

19. James SL, Bates BT, Osternig LR. Injuries to runners. Am J Sports Med 1978;6:40-50.

20. Hintermann B, Nigg BM. Pronation in runners: Implications for injuries. Sports Med 1998;26:169-176.

21. Jordan C, Barllet R. The relationship between plantar pressure distribuition and perception of confort in casual footwear (Abstract). IV Emed user meeting. Germany: 1994.

22. Manfio EF. Estudo de parâmetros antropométricos e biomecânicos do pé humano para a fabricação de calçados segundo critérios de conforto, saúde e segurança. [Dissertação de Mestrado - Programa de Pós-Graduação em Ciência do Movimento Humano]. Santa Maria (RS): Universidade Federal de Santa Maria; 1995.

23. Morag E, Cavanagh PR. Structural and functional predictors of regional peak pressures under the foot during walking. J Biomech 1999;32:359-370.

24. Cavanagh PR, Rodgers MM, Liboshi A. Pressure distribution under symton free feet during barefoot standing. Foot Ankle 1987;7:262-76.

25. Cheung RTH, Ng GYF, Chen BFC. Association of footwear with patellofemoral pain syndrome in runners. Sports Med 2006;36:199-205.

\section{Endereço para correspondência}

João Otacilio Libardoni dos Santos

Universidade do Estado de Santa Catarina

Centro de Ciências da Saúde e do Esporte

Laboratório de Biomecânica. CEFID/UDESC

Rua Pascoal Simone, 358 - Coqueiros

CEP 88080-350 - Florianópolis, SC. Brasil

E-mail: jlibardoni@yahoo.com.br 